\title{
The evolution of internal undular bores over a slope in the presence of rotation
}

\author{
By Chunxin Yuan, Roger Grimshaw and Edward Johnson
}

The large-amplitude internal waves commonly observed in the coastal ocean often take the form of unsteady undular bores. Hence, here we examine the long-time combined effect of variable topography and background rotation on the propagation of internal undular bores, using the framework of a variable-coefficient Ostrovsky equation. Since the leading waves in an internal undular bore are close to solitary waves, we first examine the evolution of a single solitary wave. Then we consider an internal undular bore, for which two methods of generation are used. One method is the matured undular bore developed from an initial shock box in the Korteweg-de Vries equation, that is the Ostrovsky equation with the rotational term omitted, and the other method is a modulated cnoidal wave solution of the same Korteweg-de Vries equation. It transpires that in the long-time model simulations, the rotational effect disintegrates the nonlinear waves into inertia-gravity waves, and then there emerge complicated interactions between these inertia-gravity waves and the modulated periodic waves of the undular bore, especially at the rear part of the undular bore. But, near the front of the undular bore nonlinear effects further modulate these waves, with the eventual emergence of nonlinear envelope wave packets.

\section{Introduction}

As demonstrated by the large amount of observational data, both in situ and from satellites, large amplitude internal waves are commonly found in coastal oceans. However, due to the complex and variable oceanic background, such as the topography, the background density distribution, and shear current, an isolated internal solitary wave is rarely observed, and instead nonlinear wave trains in a form of unsteady undular bores are more often recorded, see [1] and [2]. The typical internal undular bore consists of a series of waves connecting two different basic states and exhibiting a solitary wave train at the leading edge, see $[3,4]$. In particular a shock, that is an initial jump of fluid depth and/or velocity results in the generation of undular bores, see [5] for instance, and indeed this method is used here in some simulations.

Address for correspondence: Prof. R. Grimshaw, Department of Mathematics, University College London, London, UK, WC1E 6BT; email: r.grimshaw@ucl.ac.uk 
An other generation mechanism is when a transcritical flow passes over a localised bottom feature, such as an undersea ridge, see [6, 7, 8].

A large number of studies, see $[9,10,11,12]$ for instance, have found that the effect of the earth's rotation is significant for the propagation of internal waves over long distances. In particular [13] showed that due to the rotation, an initial largeamplitude solitary wave rapidly decays into inertia-gravity waves, and eventually the leading disturbance forms into a coherent wave packet. Although the propagation of undular bores over a topography has been widely studied, see for example [3, 4, 14], the combined impact of rotation and variable topography on the evolution of internal undular bores remains unclear, and hence this is the focus of this present paper.

In the weakly nonlinear long wave regime, the rotational effect can be described by the Ostrovsky equation, first derived by Ostrovsky, see [9], and later for waves in a channel by Grimshaw, see [15]. In the standard notation, it is

$$
\left\{A_{t}+c A_{x}+\alpha A A_{x}+\beta A_{x x x}\right\}_{x}=\gamma A .
$$

The terms inside the bracket are the Korteweg-de Vries (KdV) terms, and the rotational effect is represented by the non-local term on the right-hand side. Here $A(x, t)$ is the amplitude of the modal function $\phi(z)$, defined by

$$
\begin{aligned}
& \left\{\rho_{0}(c-u)^{2} \phi_{z}\right\}_{z}+\rho_{0} N^{2} \phi=0, \quad \text { for }-h<z<0, \\
& \phi=0 \quad \text { at } z=-h, \quad(c-u)^{2} \phi_{z}=g \phi \quad \text { at } \quad z=0
\end{aligned}
$$

which also serves to define the linear phase speed $c$. Here the background density is $\rho_{0}(z), \rho_{0} N^{2}=-g \rho_{0 z}$ and $u(z)$ is the background current. The coefficients are given by the usual expressions as for the $\mathrm{KdV}$ equation

$$
\begin{gathered}
I \alpha=3 \int_{-h}^{0} \rho_{0}(c-u)^{2} \phi_{z}^{3} d z \\
I \beta=\int_{-h}^{0} \rho_{0}(c-u)^{2} \phi^{2} d z \\
I=2 \int_{-h}^{0} \rho_{0}(c-u) \phi_{z}^{2} d z
\end{gathered}
$$

The rotational coefficient $\gamma$ is given by $\gamma=f^{2} / 2 c$ (where $f$ is the Coriolis frequency) when there is no shear flow $(u=0)$, and then we have the normal case when $\gamma \beta>0$. In contrast, when there is a shear flow it is given by

$$
I \gamma=f^{2} \int_{-h}^{0} \rho_{0} \Phi \phi_{z} d z, \quad \rho_{0}(c-u) \Phi=\rho_{0}(c-u) \phi_{z}-\left(\rho_{0} u\right)_{z} \phi
$$

and for sufficiently strong shear it is possible to have the anomalous case when $\gamma \beta<0$. This is unlikely in oceanic conditions, although that case has been looked at in $[16,17]$. However, we assume here that the normal case holds, and we take $\beta>0, \gamma>0$ for waves propagating in the positive $x$-direction. 
The Ostrovsky equation (1) can be written in an alternative form, convenient for numerical simulations,

$$
A_{t}+c A_{x}+\alpha A A_{x}+\beta A_{x x x}=\gamma B, \quad B_{x}=A \quad B=-\int_{x}^{\infty} A d x,
$$

where we assume that both $A, B \rightarrow 0$ as $x \rightarrow \infty$, since linear waves have negative group velocity. This equation has two important conservation laws,

$$
\begin{gathered}
\int_{-\infty}^{\infty} A d x=[B]_{-\infty}^{\infty}=0 \\
\frac{\partial}{\partial t} \int_{-\infty}^{\infty} A^{2} d x=0
\end{gathered}
$$

for solutions $A(x, t)$ (and then likewise $B(x, t)$ ) localised (or periodic) in $x$, which represent the conservation of mass and wave action flux respectively.

In section 2, we present the variable-coefficient Ostrovsky equation, which incorporates the effects of the slowly varying background, together with the usual rotational term. Three initial conditions are described in section 2 , followed by the corresponding numerical simulations in section 3 . We conclude in section 4.

\section{Variable-coefficient Ostrovsky equation}

\subsection{Formulation}

In the presence of a slowly varying background, specifically when the fluid depth $h$ and hydrography (if applicable) vary slowly with $x$, the Ostrovsky equation (1) is replaced by

$$
\left\{A_{t}+c A_{x}+\frac{c Q_{x}}{2 Q} A+\alpha A A_{x}+\beta A_{x x x}\right\}_{x}=\gamma A, \quad Q=c^{2} I .
$$

For simplicity here we assume that the background density $\rho_{0}(z)$ and shear flow $u(z)$ do not vary with $x$. If they did then an extra term is needed in the KdV part of the equation, see $[18,19]$. The modal equation now depends also parametrically on $x$, that is $\phi=\phi(z ; x), c=c(x)$, and hence the coefficients $\alpha, \beta, \gamma, Q$ also depend (slowly) on $x$. Since the modal equations $(2,3)$ are homogeneous, $\phi$ can be non-dimensionalised with the maximum value 1 , and the linear magnification factor $Q$ can be normalised to be unity at the initial location. It is useful to express equation (11) in non-dimensional variables based on a length scale $h_{0}$ (a typical depth), and a velocity scale $c_{0}$ (a typical linear long wave speed), so that the time scale is $t_{0}=h_{0} / c_{0}$. For instance in an ocean setting, $h_{0}=500 \mathrm{~m}$ and $c_{0}=1 \mathrm{~m} \mathrm{~s}^{-1}$ can be chosen as a typical scaling. If needed, the density $\rho_{0}$ can also be scaled with $\rho_{00}=1000 \mathrm{~kg} \mathrm{~m}^{-3}$. Then we have

$$
\begin{aligned}
& A=h_{0} \tilde{A}, \quad x=h_{0} \tilde{x}, \quad t=\frac{h_{0} \tilde{t}}{c_{0}}, \quad(c, u)=c_{0}(\tilde{c}, \tilde{u}), \\
& \alpha=\frac{c_{0} \tilde{\alpha}}{h_{0}}, \quad \beta=c_{0} h_{0}^{2} \tilde{\beta}, \quad \gamma=\frac{c_{0}}{h_{0}^{2}} \tilde{\gamma} .
\end{aligned}
$$


As a consequence, the Ostrovsky equation (11) is recovered in the ${ }^{\sim}$ variables, and also all expressions $(4-7)$ hold in the ${ }^{\sim}$ variables. Note that with $f=10^{-4} s^{-1}$ and in the absence of a background shear flow, $\tilde{\gamma} \sim f^{2} h_{0}^{2} / 2 c_{0}^{2} \sim 10^{-3} \ll 1$, while $\tilde{c}$, $\tilde{\alpha}$, $\tilde{\beta}$ are relatively order unity. As expected, in this oceanic application the rotational term can be regarded as a small perturbation to the usual KdV equation.

Formally, in terms of a small parameter $\epsilon$ used in the asymptotic derivation of equation (11), the coefficients depend on the slow variable $\hat{x}=\epsilon^{3} x$, and the corresponding amplitude is $\epsilon^{2} A(\tau, \xi)$ where $\tau=\epsilon t, \xi=\epsilon x$. It is clear that the first two terms of equation (11) are dominant terms, and hence we can transform it to an asymptotically equivalent "spatial" evolution form for $A(X, T)$,

$$
\begin{gathered}
X=\int_{x_{0}}^{x} \frac{d x}{c}-t, \quad T=\int_{x_{0}}^{x} \frac{d x}{c}, \\
\left\{A_{T}+\frac{Q_{T}}{2 Q} A+\mu A A_{X}+\lambda A_{X X X}\right\}_{X}=\sigma A, \\
\mu=\frac{\alpha}{c}, \quad \lambda=\frac{\beta}{c^{3}}, \quad \sigma=c \gamma .
\end{gathered}
$$

All terms are now of the same order, that is, $A \sim \epsilon^{2}, \partial / \partial X \sim \epsilon, \partial / \partial T \sim \epsilon^{3}$ and $\sigma \sim \epsilon^{4}$. Here the coefficients $\mu, \lambda, Q, \sigma$ depend on $T$, but note that in the absence of a background shear flow, $\sigma=f^{2} / 2$ is independent of $T$. A further exact transformation is

$$
\begin{gathered}
U=Q^{1 / 2} A, \quad\left\{U_{s}+\nu U U_{X}+U_{X X X}\right\}_{X}=\delta U, \\
\text { where } \quad \nu=\frac{\mu}{Q^{1 / 2} \lambda}, \quad \delta=\frac{\sigma}{\lambda}, \quad s=\int_{0}^{T} \lambda\left(T^{\prime}\right) d T^{\prime} .
\end{gathered}
$$

Again the Ostrovsky equation (16) can also be written in an alternative form,

$$
U_{s}+\nu U U_{X}+U_{X X X}=\delta V, \quad V_{X}=U, \quad V=-\int_{X}^{\infty} U d x,
$$

which, analogous to $(9,10)$, has two conservation laws,

$$
\begin{gathered}
\int_{-\infty}^{\infty} U d X=[V]_{-\infty}^{\infty}=0, \\
\frac{\partial}{\partial s} \int_{-\infty}^{\infty} U^{2} d x=0
\end{gathered}
$$

for solutions $U(X, s) ; V(X ; s)$ localised (or periodic) in $X$.

\subsection{Solitary wave extinction}

The linear dispersion relation of the constant-coefficient Ostrovsky equation (16) for sinusoidal waves $\sin (k X-\omega s)$ is,

$$
\omega=\frac{\delta}{k}-k^{3}
$$


and hence the phase speed and the group velocity are given by

$$
\begin{aligned}
\text { phase speed : } \quad c=\frac{\omega}{k}=\frac{\delta}{k^{2}}-k^{2} ; \\
\text { group velocity : } \quad c_{g}=\frac{d \omega}{d k}=-\frac{\delta}{k^{2}}-3 k^{2} .
\end{aligned}
$$

This dispersion relation plotted in figure 1 shows that the Ostrovsky equation is not able to support a steady solitary wave solution, as there is no gap in the linear spectrum for the phase speed $c$. This is in stark contrast to the KdV equation (that is, $\delta=0$ ) when there is a spectral gap in $c>0$ and steady solitary waves can bifurcate from $k=0$. This heuristic argument was confirmed with rigorous proofs, see [20, 21].

A KdV solitary wave with initial amplitude $a$ is extinguished in a finite time due to the radiation of inertia-gravity waves, [22], and the extinction time is given by

$$
s_{e}=\frac{1}{\delta}\left(\frac{a \nu}{12}\right)^{1 / 2}
$$

Later [13] showed that this initial KdV solitary wave is replaced by an envelope wave packet steadily propagating with a speed close to the maximum group velocity, and with the associated carrier wave number. This was confirmed experimentally, see $[21,23]$, and in further numerical simulations, see for instance [16]. These results are all for the constant-coefficient Ostrovsky equation, but recently [12] examined the combined effect of topography and rotation using a variable-coefficient Ostrovsky equation. They showed that again there is an extinction time similar to (24), but their simulations of a South China Sea transect, using both a variable-coefficient Ostrovsky equation and an ocean circulation model, were not long enough to see a total extinction. [24] examined the effects of rotation in numerical simulations of a two-layer fluid using a fully nonlinear, weakly nonhydrostatic model, and found that due to the rotation, an initial $\mathrm{KdV}$-type solitary wave decays into inertia-gravity waves, which then steepen due to nonlinearity, leading to a secondary solitary wave at the expense of the s parent wave, and this new solitary wave then experiences a similar decay. The decay and re-emergence process repeats and eventually a nearly localised wave packet emerges. A similar cycle of decay and recurrence was seen in the numerical simulations of the Ostrovsky equation ([22]), but these early simulations were not carried out for a long enough time to see the emerging wave packet.

\subsection{Undular bore asymptotic theory}

In this paper our concern is with the combined effect of topography and rotation on an undular bore, and so here we re-examine the well-known theory for a $\mathrm{KdV}$ undular bore. In the absence of rotation (that is $\delta=0$ ), the Ostrovsky equation (16) reduces to the $\mathrm{KdV}$ equation, and for a constant nonlinear coefficient $\nu$, this has the well-known periodic travelling wave solution, the cnoidal wave,

$$
\begin{gathered}
U=a\left\{b(m)+\mathrm{cn}^{2}(\varkappa \theta ; m)\right\}+d, \quad \theta=k(X-v s), \\
\text { where } \nu a=12 m \varkappa^{2} k^{2}, \quad b(m)=\frac{1-m}{m}-\frac{E(m)}{m K(m)},
\end{gathered}
$$




$$
v-\nu d=\frac{\nu a}{3}\left\{\frac{2-m}{m}-\frac{3 E(m)}{m K(m)}\right\}=4 \varkappa^{2} k^{2}\left\{2-m-\frac{3 E(m)}{K(m)}\right\} .
$$

Here $\mathrm{cn}$ is the Jacobi elliptic function of modulus $m(0<m<1), v$ is the wave speed in the transformed space and $K(m), E(m)$ are the elliptic integrals of the first and second kind respectively, defined by

$$
\begin{gathered}
\operatorname{cn}(\vartheta ; m)=\cos (\chi), \quad \vartheta=\int_{0}^{\chi} \frac{d \chi^{\prime}}{\left(1-m \sin ^{2} \chi^{\prime}\right)^{1 / 2}}, \quad 0 \leq \chi \leq \frac{\pi}{2}, \\
K(m)=\int_{0}^{\pi / 2} \frac{d \chi}{\left(1-m \sin ^{2} \chi\right)^{1 / 2}}, \quad E(m)=\int_{0}^{\pi / 2}\left(1-m \sin ^{2} \chi\right)^{1 / 2} d \chi .
\end{gathered}
$$

The cnoidal wave $U(\theta)(25)$ is periodic both spatially and temporally, manifested by requiring that it be periodic in $\theta$ with a period of $2 \pi$. Then the wavenumber $\varkappa=K(m) / \pi$, while the spatial period is $2 \pi / k$. The (trough-to-crest) amplitude is $a$ and the mean value of $U$ over one period is $d$. This solution family has three independent parameters, say $k, m, d$. There are two important limiting cases. One is when the modulus $m \rightarrow 1$, and then this becomes a solitary wave train, since then $b \rightarrow 0$ and $\operatorname{cn}(\vartheta) \rightarrow \operatorname{sech}(\vartheta)$, while $\varkappa \rightarrow \infty, k \rightarrow 0$ with $\varkappa k=\varpi$ fixed. The other case is when $m \rightarrow 0, b \rightarrow-1 / 2, \varkappa \rightarrow 1 / 2, \operatorname{cn}(\vartheta) \rightarrow \cos (\vartheta)$, and it reduces to a sinusoidal wave $(a / 2) \cos (\theta)$ of small amplitude $a \sim m$ and wavenumber $k$.

Whitham modulation theory can now be used to construct an undular bore by allowing this cnoidal wave to vary slowly with $s, X$, that is the wavenumber $k$, modulus $m$ and mean level $d$ vary slowly with $s, X$. The Whitham modulation equations describing this variation can be obtained by averaging conservation laws, see [25, 26], or by exploiting the integrability of the constant-coefficient $\mathrm{KdV}$ equation, see [27] for example. When nonlinear coefficient $\nu$ in (16) is a constant, we note especially a similarity solution to the Whitham modulation equations which describes an undular bore evolving from an initial step of height $U_{0}$ where $\nu U_{0}>0$, see $[25,28]$,

$$
\begin{gathered}
\frac{X}{s}=\frac{\nu U_{0}}{3}\left\{1+m-\frac{2 m(1-m) K(m)}{E(m)-(1-m) K(m)}\right\}, \quad-\nu U_{0}<\frac{X}{s}<\frac{2 \nu U_{0}}{3}, \\
a=2 U_{0} m, \quad d=U_{0}\left\{m-1+\frac{2 E(m)}{K m}\right\}, \quad \nu U_{0}=6 \varkappa^{2} k^{2}, \quad v=\frac{\nu U_{0}}{3}\{1+m\} .
\end{gathered}
$$

Note that

$$
X-v s=\frac{\nu U_{0} s}{3}\left\{-\frac{2 m(1-m) K(m)}{E(m)-(1-m) K(m)}\right\}
$$

is negative for all $X, s$. This describes a wave train connecting a zero level at the front where $m \rightarrow 1$ to a mean level $U_{0}$ at the rear where $m \rightarrow 0$. At the front the leading wave is a solitary wave of amplitude $2 U_{0}$, while at the rear the waves are linear sinusoidal waves with a very small amplitude, and some intermediate waves whose nonlinearity (indicated by $m$ ) is decreasing from the front to rear exist between these two edges. Note that with the evolution, the whole undular bore is expanding with "time" $s$, but nevertheless it is always confined in a range $-\nu U_{0} s<X<2 \nu U_{0} s / 3$. 
Following $[4,14,29]$ it is now useful to examine the solitary wave train at the leading edge of the undular bore, formally obtained by taking the limit $m \rightarrow 1$. In this limit, the three Whitham modulation equations uncouple and can be explicitly solved. Then it is found that the deformation of a solitary wave train in a non-rotating variable medium $\nu=\nu(s), \delta=0$ can be classified into two scenarios, depending on whether there is a polarity change, that is $\nu$ changes sign, or not. Each of these are now well understood, see the reviews in [7, 23, 30]. The outcome for a solitary wave train is that the amplitude $a$ of the leading wave in the solitary wave train varies according to the law $a^{3} \propto \nu$, while the amplitudes in the solitary wave train, relative to this adiabatic deformation, have a similarity structure proportional to $X / s$. This scenario holds provided $\nu \neq 0$. But if there is a change of polarity, that is there is a critical point where $\nu$ passes through zero and changes sign. After the critical point a rarefaction wave with a similarity structure and of opposite polarity to the solitary waves emerges, terminated by an undular bore. For an undular bore, these descriptions can be applied to the leading solitary wave train in an undular bore, but a full description using Whitham modulation theory in a variable medium cannot be obtained, due to the development of non-adiabatic behaviour in the region between the quasi-periodic undular bore wave train, and the solitary wave train emitted ahead of this structure, see [4]. Taking rotation into account as well would seem to be beyond current theoretical capacity, but we note that [17] derived the Whitham modulation equations for the Ostrovsky equation, albeit for constant coefficients and in the weak rotation limit (two orders smaller).

\section{Numerical simulations}

\subsection{Set-up and initial conditions}

Since there is no satisfactory theory which can describe the combined effects of topography and rotation on internal undular bores, we resort here to numerical simulations of the variable-coefficient Ostrovsky equation. The numerical simulations are carried out in the transformed space, using the Ostrovsky equation (16). A pseudo-spectral method based on a Fourier interpolant is used in the spatial domain, while a classical Runge-Kutta fourth-order method, together with a very fine time step, provides an accurate iteration in the time domain. By construction, the solution in $X$ direction is periodic, thus two sponge layers are implemented at two ends of the domain to avoid the radiated waves re-entering the calculation area.

As already mentioned, under real oceanic circumstances, normally the effect of background rotation is quite weak, thus only when waves propagate a long distance, or equivalently, with a time scale exceeding several inertial periods, it does become potentially significant. Thus. to obtain a long time solution to the Ostrovsky equation, a scaling transformation is used,

$$
s=s_{0} \bar{s}, \quad X=X_{0} \bar{X}, \quad U=U_{0} \bar{U}, \quad s_{0}=X_{0}^{3}, \quad \delta X_{0}^{4}=\bar{\delta}, \quad \nu U_{0} X_{0}^{2}=\bar{\nu},
$$

where two independent parameters $X_{0}$ and $U_{0}$ can be chosen to scale both the new nonlinear coefficient $\bar{\nu}$ and new rotation coefficient $\bar{\delta}$ to be of order unity. The Ostrovsky equation (16) and (18) retain their expressions in the new ${ }^{-}$variables and 
henceforth the ${ }^{\sim}$ symbols are omitted. Note that then the rescaled initial condition $\bar{U}$ needs to be quite large, as $U_{0}=\sqrt{\delta} / \nu$ is typically quite small. In terms of the original coefficients in (11), $U_{0}=\sqrt{\gamma \beta} / \alpha$ and further using the non-dimensional analysis introduced by the expression (12), $U_{0}=h_{0} \sqrt{\tilde{\gamma} \tilde{\beta}} / \tilde{\alpha} \sim 10^{-1}$.

Since the available theory and the simulations are to be applied to real oceanic conditions, in practice we should evaluate all coefficients of the Ostrovsky equation through the expressions $(2-7)$, and then use the series of transformations described in section 1 to achieve the convenient form of (16), together with the corresponding new coefficients, and finally the scaling transformation (33) is used to facilitate the attainment of a long-time solution. However, since the goal of this paper is to detect the underlying dynamics of internal undular bores propagating over a variable bottom topography under the influence of rotation, it is sufficient to use an idealised process model. Hence, we choose the nonlinear coefficient $\nu=\nu(s)$ as a function of $s$ only varying monotonically from $\nu=1$ at $s=0$ to some constant value $\nu=\nu_{a}$ for $s \geq s_{a}$. Specifically,

$$
\nu=1+\left(\nu_{a}-1\right) \tanh (K s),
$$

where $K, s_{a}, K s_{a} \gg 1$ are chosen so that $\nu$ varies smoothly and slowly from 1 at $s=0$ to $\nu_{a}$ at $s=s_{a}$. There are two main scenarios, either $\nu_{a}>0$ for propagation up a slope, or $\nu_{a}<0$ for propagation up a slope and through a point of polarity change. Likewise, the rotation coefficient $\delta=\delta(s)$ should be chosen to be initially quite small, $\delta=0.5$ here, corresponding to the strong dispersion in deep water, and then increase to a constant value $\delta_{a}>0.5$, corresponding to propagation up a slope. A suitable choice is

$$
\delta=0.5+\left(\delta_{a}-0.5\right) \tanh (K s),
$$

so that $\delta$ increases from 0.5 to a constant value $\delta_{a}$ for $s \geq s_{a}$.

The initial condition is $U(X, s=0)=U_{i c}(X)+D(X)$ where $D(X)$ (see (42) below) is a pedestal needed to ensure that the mass constraint (19) is satisfied at the initial value $s=0$ and $U_{i c}(X)$ is either (1) a KdV solitary wave, or (2) a box of height $U_{0}>0$ which in the absence of rotation and variable topography would generate an undular bore followed by a rarefaction wave, or (3) a modulated cnoidal wave representation of an undular bore in the constant coefficient $\mathrm{KdV}$ equation evolving from a step of height $U_{0}>0$ at time $s=-s_{1}$, given by $(30-32)$ with $s$ replaced by $\left(s+s_{1}\right)$ and then evaluated at $s=0$ when $\nu=1$,

$$
\begin{aligned}
& (1): U_{i c}(X)=a \operatorname{sech}^{2}(\varkappa X), \quad a=12 \varkappa^{2}, \\
& (2): U_{i c}(X)=U_{0} \operatorname{ENV}(X), \\
& \operatorname{ENV}(X)=\frac{1}{2}\{\tanh \Gamma(X+L)-\tanh \Gamma(X-L)\}, \\
& (3): U_{i c}(X)=U_{0} \operatorname{ENV}(X)\left\{2 m \mathrm{cn}^{2}\left(\varkappa\left(X-v s_{1}\right) ; m\right)+1-m\right\}, \\
& -U_{0} s_{1}<X<\frac{2 U_{0} s_{1}}{3}, \quad U_{0}=6 \varkappa^{2} k^{2}, \quad v=\frac{U_{0}}{3}\{1+m\}, \\
& \quad X=\frac{U_{0} s_{1}}{3}\left\{1+m-\frac{2 m(1-m) K(m)}{E(m)-(1-m) K(m)}\right\} .
\end{aligned}
$$


In case (1) the evolving solitary wave has a time scale of $(\varkappa V)^{-1}$ where the speed $V=4 \varkappa^{2}$, and so to be slowly varying we choose $K \ll \varkappa V=4 \varkappa^{3}$ in expression (34) and (35). In case (2) the envelope $\operatorname{ENV}(X)$ is chosen to be very close to a box of height 1 , and of very long length $2 L$, that is to say $K^{-1} \ll 1 \ll 2 L$. With a constant $\nu=1$ and $U_{0}>0$ in the $\mathrm{KdV}$ regime, the front end of the box is then expected to generate an undular bore in the subsequent evolution. Since the leading wave in the undular bore is a solitary wave of amplitude $2 U_{0}$, so again $K$ should be small enough to warrant $K \ll 4 \varkappa^{3}$, where here $U_{0}=6 \varkappa^{2}$. At the same time, the rear end of the box will generate a rarefaction wave, and some model time later, the calculation will be cut off and the matured undular bore is now ready to be put into the Ostrovsky equation. In case (3) the asymptotic solution is similar to the undular bore generated in case (2), but we can now control the parameters more effectively. The initial undular bore at $s=0$ occupies the domain $-U_{0} s_{1}<X<2 U_{0} s_{1} / 3$. We choose $s_{1}$ to make sure this domain has length $L_{u b}=5 U_{0} s_{1} / 3 \gg 1$. The envelope $\operatorname{ENV}(X)$ has a similar structure as that in case (2), but a constraint is put on, that is the front end of the box is placed precisely at the front end of the undular bore $X=2 U_{0} s_{1} / 3$, while the rear end is chosen far away from the rear end of the bore, which means the initial undular bore is contained in the box with $L>L_{u b}$. Analogous to case (2), the leading wave is a solitary wave of amplitude $2 U_{0}$ and thus again $K$ should be small, $K \ll 4 \varkappa^{3}$ where here $U_{0}=6 \varkappa^{2} k^{2}$. Note that the wavelength $2 \pi / k$ is a free parameter. The pedestal $D(X)$ is represented in these three respective cases as

$$
\begin{array}{ll}
\text { (1) : } & \int_{-\infty}^{\infty} D(X) d X=-\int_{-\infty}^{\infty} a \operatorname{sech}^{2}(\varkappa X) d X=-\frac{2 a}{\varkappa}=-24 \varkappa, \\
\text { (2) : } & \int_{-\infty}^{\infty} D(X) d X=-a \int_{-\infty}^{\infty} \operatorname{ENV}(X) d X=-2 a L, \\
\text { (3) : } & \int_{-\infty}^{\infty} D(X) d X \approx-a L,
\end{array}
$$

such that the initial mass is zero. In case (3) we have estimated the integral as approximately half that of case (2) since the initial step is located at the halfway point of the envelope $\operatorname{ENV}(X)$. For a numerical domain of total length $2 L_{n}$, a simple choice is (1) $D=-12 \varkappa / L_{n}$, (2) $D=-L / L_{n}$ and (3) $D=-0.5 L / L_{n}$. However a better choice to avoid end effects is

$$
D(X)=\frac{D_{0}}{2}\left\{\tanh \left(\frac{X+L_{e}}{L_{w}}\right)-\tanh \left(\frac{X-L_{e}}{L_{w}}\right)\right\}, \quad \int_{-\infty}^{\infty} D(X) d X=2 D_{0} L_{e}
$$

where $L_{e}=L_{n} / 2, L_{w}=L_{n} / 4$. To keep the pedestal small, we need $\left|D_{0}\right| \ll a$, or $D_{0} \ll U_{0}$, that is, $\varkappa L_{e} \gg 1$ or $L_{e} \gg L$.

\subsection{Numerical results}

First we examine the evolution of an initial KdV solitary wave, case (1), the same as that considered in [13] for a constant environment, but now for a variable medium with $\nu=\nu(s)$ and $\delta=\delta(s)$. The outcome is shown in figure 2 and we see that the outcome is quite similar to the constant-coefficient case of [13]. Eventually the 
initial solitary wave is replaced by a wave packet followed by trailing waves. The rotational effect appears to be dominant in this circumstance, with the nonlinear effect partly suppressed, as the variation of the nonlinear coefficient $\nu$, specifically the occurrence of a polarity change or not, does not seem to make much impact on the subsequent evolution. Moreover in both cases (see the middle and right panels) the amplitude of the resultant wave packet is almost the same, although when $\nu_{a}=-1$ (polarity change), the envelope tends to lie below the zero level more than when $\nu_{a}=0.2$ (no polarity change), which indicates some small influence of nonlinearity. We recall that in the KdV regime, see [7, 14, 30], when there is no polarity change ( $\nu$ changes from 1 to 0.2 ), then to conserve the wave action flux, the adiabatic law shows that the amplitude of the leading solitary wave $a$ behaves as $a \propto \nu^{1 / 3}$, but at the same time, conservation of mass results in a trailing shelf which has an amplitude at the solitary wave location proportional to $\nu^{-8 / 3} \nu_{s}$ (normally it is approximately one order smaller than $\nu^{1 / 3}$ ). In contrast, the situation is different in the case with a polarity change, where the leading solitary wave and the trailing shelf have comparable amplitudes near the critical point where $\nu=0$, and normally they both are smaller than their counterparts without a polarity change. Hence, in the Ostrovsky equation, the combined effect of rotation and nonlinearity is exerted over the solitary wave, and one of expected evolution scenarios is that when $\nu_{a}=0.2$, the outcome is characterised by a larger amplitude of the envelope and a smaller number of waves contained in the trailing shelf, in contrast to the case with $\nu_{a}=-1$. Note that here the extinction time $s_{e}(24)$ is order unity. Since the the model run-time is $s_{a}=100$, then $s_{e} \sim 1$ is relatively too short to take the variation of the nonlinear term into account, and hence leads to the dominant rotational effect.

The initial internal undular bore is generated for convenience from the evolution of a long box (37) in the framework of KdV equation, see figure 3. Moreover, in the full Euler equations, this initial box can also be used to generate an undular bore (not shown here). This resultant undular bore can be theoretically described by the modulated cnoidal wave (38). The behaviour of undular bores propagating over a variable topography was studied in [4] in a water wave context and [14] in a variable-coefficient $\mathrm{KdV}$ model. The effect of the slowly varying topography is the generation of a solitary wave train ahead of the main undular bore, and these two parts are connected where the rear of the solitary wave train interacts with the main undular bore, forming a two-phase modulated wave train. When there is a polarity change, on passage through the critical point (nonlinear coeffcient $\nu=0$ ), the leading solitary wave train in the undular bore is not able to retain its shape and is gradually replaced by developing rarefaction waves supporting emerging solitary waves of the opposite polarity, while the rear near-linear periodic waves hold their shapes, but with a rotational effect is jointly taken into account, the outcome becomes more complicated, see figures 3-5. In general the internal undular bore decays into several wave packets, accompanied by a few residual waves. More specifically and heuristically, the leading nonlinear waves behave like solitary waves, as shown in figure 2 , but note that then this is followed by inevitable complicated interactions between the radiated inertial-gravity waves and also with the original rear periodic waves, some of which form wave packets propagating coherently. Eventually, some nearly localized wave packets emerge, each one consisting of a long-wave envelope through 
which shorter, faster waves propagate. Furthermore, depending on the parameter $s_{1}$ in the initial condition, which determines the number of waves contained in the initial bore, a different number of wave packets finally appear after a long-time evolution. It seems that the more waves there are in the initial bore, then more wave packets are formed, see figure 4 and 5 (cf. figure 3). Unlike the aforementioned cases of a solitary wave, for an undular bore, the variation of nonlinear effect plays a crucial role in the evolution. Comparing the cases of $\nu_{a}=0.2$ with that of $\nu_{a}=-1$ in figures 3-5, it is clear that the value of $\nu_{a}$ can influence the formation of eventual wave packets to some finite degree. First, when $\nu_{a}=-1$, the amplitude of the wave envelope, is larger than that when $\nu_{a}=0.2$, and also it tends to lie further below the zero level, which can be partially attributed to the nonlinear steepening. The number of wave packets eventually formed and the length scale of each wave packet are related to nonlinear effects, that is, there are more wave packets but each with a shorter length scale when $\nu_{a}=-1$ than when $\nu_{a}=0.2$.

\section{Conclusion}

Our focus on this paper is how an internal undular bore behaves in a long-time limit when the combined effect of topography and background rotation are both taken into account. Since the leading part of an undular bore is composed of a solitary wave train, an initial condition consisting of a single solitary wave is first examined. As found in previous studies, such as $[13,21]$, due to the rotation, the initial solitary wave decays through the generation of inertia-gravity waves and is completely extinguished on a time scale of $s_{e}=\sqrt{a \nu / 12} / \delta$. In our long-time model simulation, eventually a coherent envelope wave packet emerges to replace the initial wave. Compared with the set-up of a constant environment in $[13,21]$, here we consider a variable background environment to simulate internal waves propagating shorewards. We find that the rotational effect is dominant, while nonlinear effects slightly modulate the waveform and amplitude. We note that [12] investigated a similar problem, where they applied the variable-coefficient Ostrovsky equation to a transect in the South China Sea. They showed that the combined effect of shoaling and rotation is to induce a secondary trailing wave packet. Because their model-run time was not very long, the difference with our presented results can be attributed to our much longer model run-time, as here the extinction time $s_{e} \sim 1$, while we run the model for $s_{a}=100 \gg s_{e}$.

If only the topographic effect is considered, then depending on whether the waves pass through a critical point (the nonlinear coefficient $\nu=0$ ) or not, the evolution scenarios of an undular bore can be quite different, see [4, 14] for details. But, when the rotational effect is also included, the evolution of inertia-gravity waves, resulting in a complicated interactions between these radiated waves with each other, and with the rear part of the undular bore, while at the front of the undular bore a few envelope wave packets form and propagate coherently. The nonlinear effects can influence the eventual emergence of these wave packets, manifested with a larger envelope amplitude, more wave packets and a shorter envelope length scale when there is a passage through the critical point $\left(\nu_{a}=-1\right)$ than when there is no polarity change $\left(\nu_{a}=0.2\right)$. 
We emphasise that the present simulations are for a process model, using the transformed variable-coefficient Ostrovsky equation (16). To apply our results to actual oceanic states requires a transformation back to the physical variables using the expressions in $(13,16,17)$. However, these change only the amplitude and time scales, and do not change the essential dynamics revealed here. But we caution that, as in the study in [2] of several SAR images, and in the MITgcm simulations in [12] of a South China Sea (SCS) transect, in order for rotational effects to become evident, the actual time scale for the evolution of internal solitary waves from the source to the continental shelf should be at least comparable with the extinction time (24). For instance in [2] there is an examination of two different transects of the SCS where there have been many observations and much research on internal waves. They found extinction times in the range $3-6$ days with corresponding linear phase speeds of the order of $2.5 \mathrm{~m} \mathrm{~s}^{-1}$. With a propagation distance of order $1000 \mathrm{~km}$ this implies a propagation time of the order of 4.5 days, comparable with the estimated extinction times. This is in agreement with the observational studies in [31,32] of the evolution of the internal tide into internal solitary waves in the deep basin of the SCS, where they found that rotational effects were quite significant.

\section{Acknowledgements}

Chunxin Yuan was supported by the Chinese Scholarship Council and UCL Dean's prize. Roger Grimshaw was supported by the Leverhulme Trust through the award of a Leverhulme Emeritus Fellowship.

\section{References}

1. P. E. La Violette, D. R. Johnson, and D. A. Brooks, Sun-glitter photographs of georges bank and the gulf of maine from the space shuttle, Oceanography 3:43-49 (1990).

2. R. Grimshaw, J. C. Da Silva, and J. M. Magalhaes, Modelling and observations of oceanic nonlinear internal wave packets affected by the Earth's rotation, Ocean Modelling 116:146-158 (2017).

3. G. A. El, R. H. J. Grimshaw, and A. M. Kamchatnov, Evolution of solitary waves and undular bores in shallow-water flows over a gradual slope with bottom friction, Journal of Fluid Mechanics 585:213-244 (2007).

4. G. EL, R. H. Grimshaw, and W. K. Tiong, Transformation of a shoaling undular bore, Journal of Fluid Mechanics 709:371-395 (2012).

5. G. A. El, R. H. J. Grimshaw, and N. F. Smyth, Unsteady undular bores in fully nonlinear shallow-water theory, Physics of Fluids 18:027104 (2006).

6. P. G. Baines, Topographic effects in stratified flows, Cambridge University Press, 1998.

7. R. Grimshaw, Internal solitary waves in a variable medium, GAMM-Mitteilungen 30:96-109 (2007).

8. G. A. El, R. H. J. Grimshaw, and N. F. Smyth, Transcritical shallow-water flow past topography: finite-amplitude theory, Journal of Fluid Mechanics 640:187 (2009).

9. L. A. Ostrovsky, Nonlinear internal waves in a rotating ocean, Oceanology 18:119-125 (1978).

10. T. Gerkema, A unified model for the generation and fission of internal tides in a rotating ocean, Journal of Marine Research 54:421-450 (1996).

11. K. R. Helfrich and R. H. J. Grimshaw, Nonlinear disintegration of the internal tide, Journal of Physical Oceanography 38:686-701 (2008). 
12. R. Grimshaw, C. Guo, K. Helfrich, and V. Vlasenko, Combined effect of rotation and topography on shoaling oceanic internal solitary waves, Journal of Physical Oceanography 44:1116-1132 (2014).

13. R. Grimshaw and K. Helfrich, Long-time solutions of the Ostrovsky equation, Studies in Applied Mathematics 121:71-88 (2008).

14. R. GRImshaw and C. YUAN, The propagation of internal undular bores over variable topography, Physica D: Nonlinear Phenomena 333:200-207 (2016).

15. R. Grimshaw, Evolution equations for weakly nonlinear, long internal waves in a rotating fluid, Studies in Applied Mathematics 73:1-33 (1985).

16. R. Grimshaw, Y. Stepanyants, and A. Alias, Formation of wave packets in the Ostrovsky equation for both normal and anomalous dispersion, Proc. R. Soc. A 472:20150416 (2016).

17. A. J. Whitfield and E. R. Johnson, Whitham modulation theory for the Ostrovsky equation, Proc. R. Soc. A 473:20160709 (2017).

18. X. Zhou and R. Grimshaw, The effect of variable currents on internal solitary waves, Dynamics of Atmospheres and Oceans 14:17-39 (1989).

19. Z. Liu, R. Grimshaw, and E. Johnson, Internal solitary waves propagating through variable background hydrology and currents, Ocean Modelling 116:134-145 (2017).

20. V. N. Galkin and Y. A. Stepanyants, On the existence of stationary solitary waves in a rotating fluid, J. Appl. Maths. Mech. 55:939-943 (1991).

21. R. Grimshaw and K. Helfrich, The effect of rotation on internal solitary waves, IMA J. Appl. Math. 77:326-339 (2012).

22. R. Grimshaw, J.-M. He, and L. Ostrovsky, Terminal damping of a solitary wave due to radiation in rotational systems, Stud. Appl. Math. 101:197-210 (1998).

23. R. Grimshaw, Change of polarity for periodic waves in the variable-coefficient Korteweg-de Vries equation, Studies in Applied Mathematics 134:363-371 (2015).

24. K. R. Helfrich, Decay and return of internal solitary waves with rotation, Physics of fluids 19:026601 (2007).

25. G. B. Whitham, Non-linear dispersive waves, Proceedings of the Royal Society of London A: Mathematical, Physical and Engineering Sciences, vol. 283 The Royal Society, 1965 pp. $238-261$.

26. G. B. Whitham, Linear and nonlinear waves, vol. 42, John Wiley \& Sons, 2011.

27. A. M. Kamchatnov, Nonlinear Periodic Waves and Their Modulations: An Introductory Course, World Scientific, 2000.

28. A. Gurevich and L. Pitayevsky, Nonstationary structure of a collisionless shock wave, Soviet Journal of Experimental and Theoretical Physics 38:590-604 (1974).

29. R. Grimshaw and C. YuAn, Internal undular bores in the coastal ocean, The Ocean in Motion, ed. M. Velarde, R. Taraknov, A. Marchenko, Springer Oceanography Series, 2017 pp. ??

30. R. Grimshaw, E. Pelinovsky, T. Talipova, and O. Kurkina, Internal solitary waves: propagation, deformation and disintegration, Nonlinear Processes in Geophysics 17:633 (2010).

31. D. FArmer, Q. Li, and J.-H. PARK, Internal wave observations in the South China Sea: The role of rotation and non-linearity, Atmosphere-Ocean 47:267-280 (2009).

32. Q. LI and F. D. M., The generation and evolution of nonlinear internal waves in the deep basin of the South China Sea, Journal of Physical Oceanography 41:1345-1363 (2011).

Department of Mathematics, University College London, London, UK, WC1E 6BT 


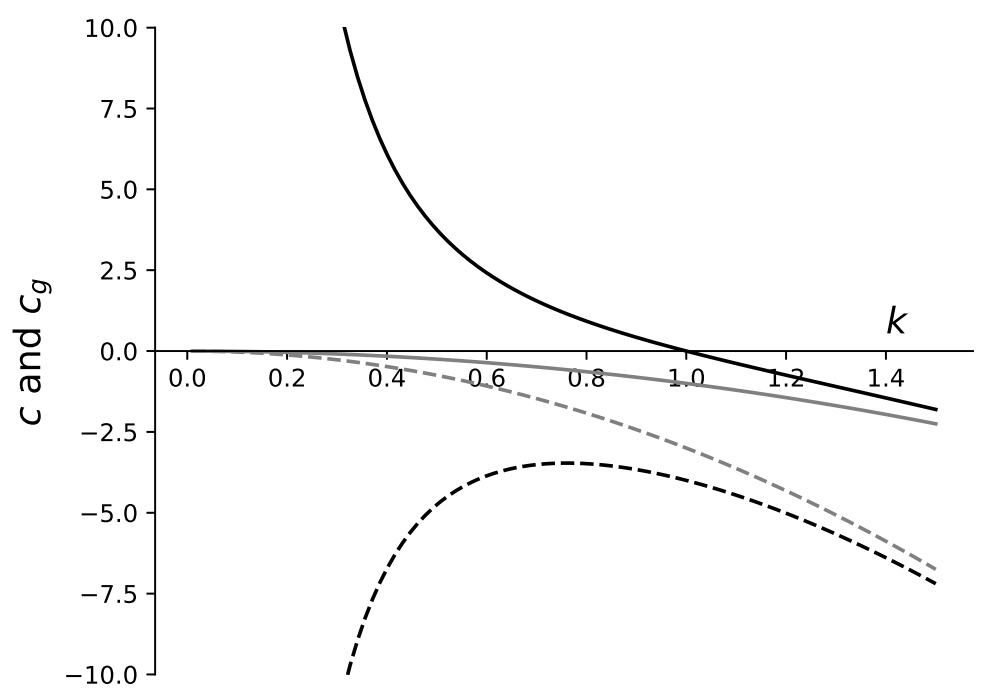

Figure 1. The phase speed $c$ and group velocity $c_{g}$ in $(22,23)$ are shown by solid and dashed lines respectively. The Ostrovksy equation $(\delta=1$ in equation (16)) is in dark, whereas the $\mathrm{KdV}$ equation $(\delta=0$ in $(16))$ is in grey.

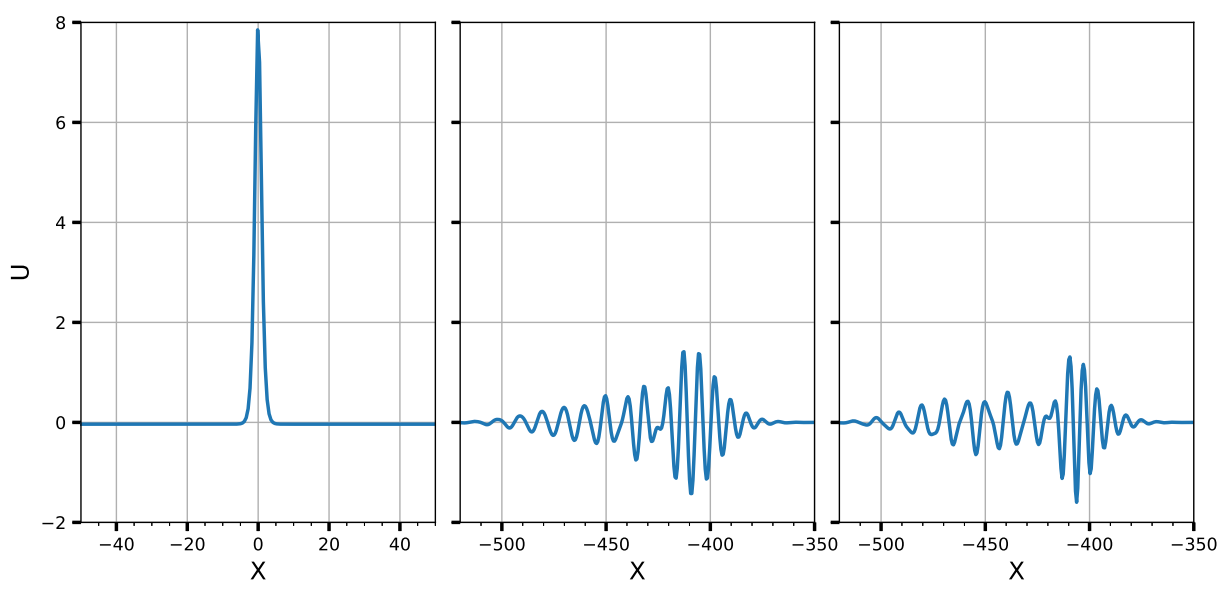

Figure 2. A simulation of the Ostrovsky equation (16) for the internal solitary wave initial condition (36). The left panel is at $s=0$ with $a=8$ when $\nu=1$ and $\delta=0.5$; the middle panel is the case without a polarity change at $s_{a}=100$ when $\nu_{a}=0.2$ and $\delta_{a}=1.5$; the right panel is for the case of polarity change, at $s_{a}=100$ when $\nu_{a}=-1$ and $\delta_{a}=1.5$. In both cases, $K=0.05$ in (34) and (35), so that $K s_{a}=5$. 

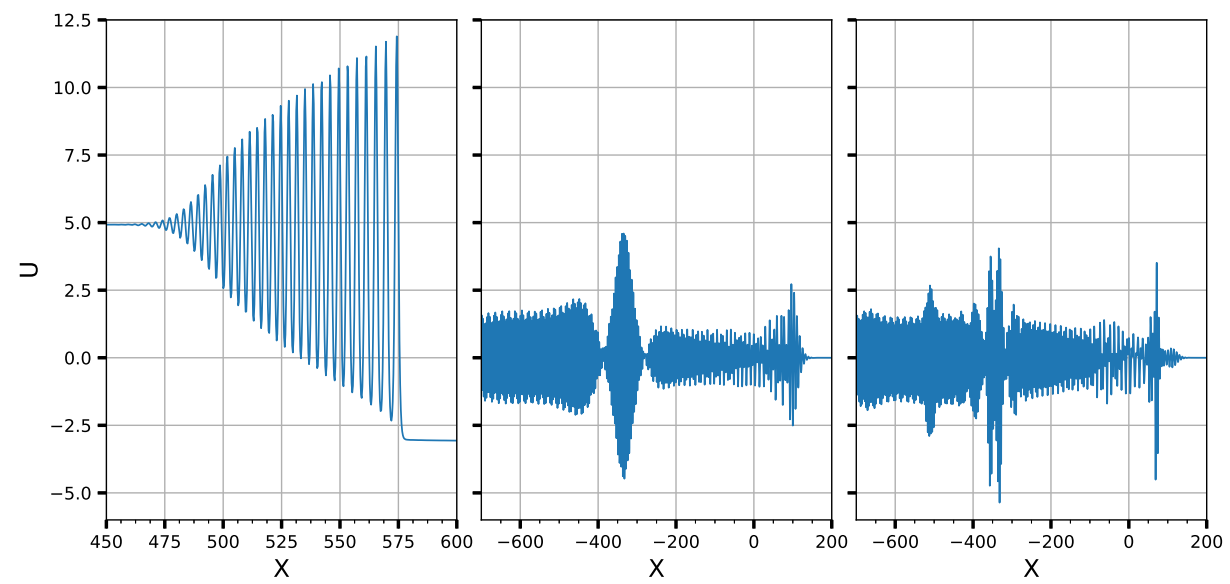

Figure 3. A simulation of the Ostrovsky equation (16) for the box initial condition (37). The left panel is the matured undular bore developed in the KdV equation with a constant coefficient $\nu=1$ starting from the initial box with $U_{0}=8$ for a run-time duration $s=20$. Then afterwards, this undular bore is used as the input to the Ostrovsky equation in which a combined effect of varying rotation and nonlinearity is considered, as given in (34) and (35). The middle panel is at $s_{a}=120$ (the origin $s=0$ of time domain is reset in the Ostrovsky equation) when $\nu_{a}=0.2$ and $\delta_{a}=1.5$; the right panel is for the case of polarity change, at $s_{a}=120$ when $\nu_{a}=-1$ and $\delta_{a}=1.5$. In both cases, $K=0.03$ in (34) and (35), so that $K s_{a}=3.6$. 

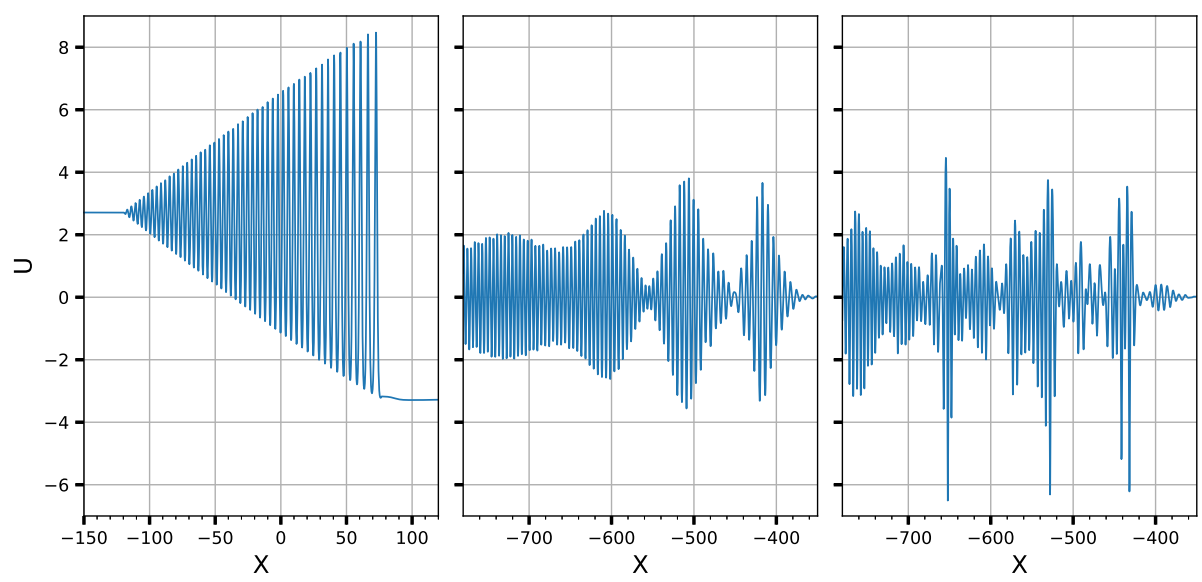

Figure 4. A simulation of the Ostrovsky equation (16) for the initial condition (38). The left panel is at $s=0$ with $U_{0}=6, s_{1}=20$ and wavenumber $k=1$ when $\nu=1$ and $\delta=0.5$; the middle panel is the case without a polarity change at $s_{a}=120$ when $\nu_{a}=0.2$ and $\delta_{a}=1.5$; the right panel is for the case of polarity change, at $s_{a}=120$ when $\nu_{a}=-1$ and $\delta_{a}=1.5$. In both cases, $K=0.03$ in (34) and (35), so that $K s_{a}=3.6$.
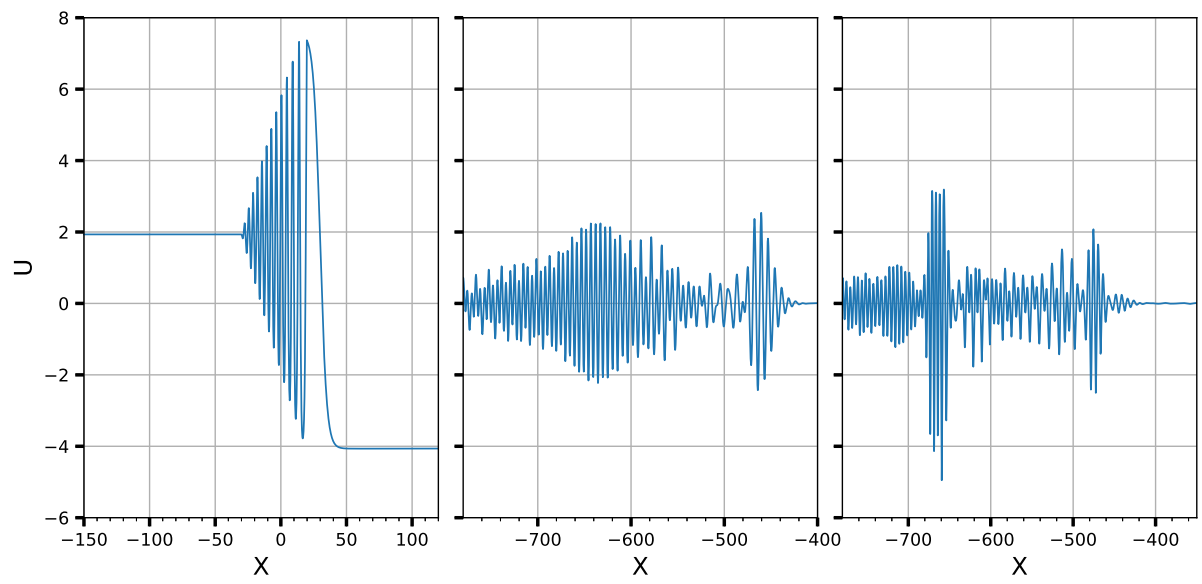

Figure 5. The same as in figure 4 , apart from that $s_{1}=5$. 